\title{
Energy Gap, Microwave-Assisted Tunneling, and Josephson Steps in Thin-Film Weak Links at 63 and $302 \mathrm{GHz}$
}

\author{
Kofoed, Bent; Særmark, Knud
}

Published in:

Physical Review Letters

Link to article, DOI:

10.1103/PhysRevLett.31.1124

Publication date:

1973

Document Version

Publisher's PDF, also known as Version of record

Link back to DTU Orbit

Citation (APA):

Kofoed, B., \& Særmark, K. (1973). Energy Gap, Microwave-Assisted Tunneling, and Josephson Steps in ThinFilm Weak Links at 63 and 302 GHz. Physical Review Letters, 31(18), 1124-1126.

https://doi.org/10.1103/PhysRevLett.31.1124

\section{General rights}

Copyright and moral rights for the publications made accessible in the public portal are retained by the authors and/or other copyright owners and it is a condition of accessing publications that users recognise and abide by the legal requirements associated with these rights.

- Users may download and print one copy of any publication from the public portal for the purpose of private study or research.

- You may not further distribute the material or use it for any profit-making activity or commercial gain

- You may freely distribute the URL identifying the publication in the public portal 


\title{
Energy Gap, Microwave-Assisted Tunneling, and Josephson Steps in Thin-Film Weak Links at 63 and $302 \mathrm{GHz}$
}

\author{
B. Kofoed and K. Saermark \\ Physics Laboratory I, The Technical University of Denmark, DK-2800 Lyngby, Denmark
} (Received 17 July 1973)

\begin{abstract}
We present experimental evidence for the occurrence of energy-gap structure and microwave-assisted tunneling in the $I V$ curves for superconducting thin-film weak links. From measurements of the power and the temperature dependence of the Josephson steps we argue that also the Riedel peak is observable in weak links.
\end{abstract}

The occurrence of structure caused by the superconducting energy gap, subharmonics of the energy gap, microwave-assisted tunneling including the satellite structure connected with the subharmonics of the gap, ${ }^{1-3}$ and the Josephson effect $^{4}$ in tunnel junctions and point contacts is by now a well-established fact. Also the quasiparticle-pair interference $\cos \varphi$ term has been observed in tunnel junctions. ${ }^{5}$ In thin-film weak links, on the other hand, only the Josephson steps appear to be well established through the obser vations of Gregers-Hansen et al. ${ }^{6}$ at 3 and $9 \mathrm{GHz}$ close to $T_{c}$ and through the Dayem effect. ${ }^{7} \mathrm{Re}$ cently the $\cos \varphi$ term may have been observed. ${ }^{8}$ The thin-film weak links used by Dayem were prepared by an evaporation technique, whereas Gregers-Hansen et al. used a razor-blade technique. In the latter case the dimensions are of the order $0.5 \mu \mathrm{m}$ wide by $0.2 \mu \mathrm{m}$ long.

In the present note we report on measurements on thin-film weak links fabricated in a novel way which will be described in detail elsewhere. The shape of our weak links may be seen in the inset in Fig. 1. The resistance of a weak link is of the order 300-600 $\Omega$ at room temperature, and below $T_{c}$ the normal resistance is of the order of 50-100 $\Omega$. The thickness of the film is about $1000 \AA$. During the measurements the weak link is mounted outside, but very close to, the end of a 70-GHz wave guide in a glass cryostat equipped with magnetic shielding. The 70V10 OKI klystron and the 300-GHz Carcinotron used deliver 25 and $50 \mathrm{~mW}$, respectively. The electronic equipment measures the $I V$ characteristic and the dynamic resistance $d V / d I$ or the dynamic conductance $d I /$ $d V$ as function of the bias voltage.

Although our measurements include several weak links of In and $\mathrm{Sn}$, and some of $\mathrm{Pb}$, we here discuss mainly the results obtained using In, as the results for $\mathrm{Sn}$ and $\mathrm{Pb}$ are qualitatively similar. The structures in the $I V$ characteristics reported in this Letter have always been seen when looked for. Briefly, we have observed a gap structure, microwave-assisted tunneling, and Josephson steps, and we have examined in some cases the power and temperature dependences of these effects.

(1) Gap structure.-In Fig. 1 the topmost trace shows the $I V$ characteristic for a thin-film In weak link without incident microwaves and at a temperature of $1.58 \mathrm{~K}$, i.e., $t=0.466$. The bump on the curve, indicated by the arrow $a$, occurs at a voltage which corresponds well with the energy gap value $2 \Delta / e=1.02 \mathrm{mV}$ for In at this temperature. The same bump occurs also on both the middle and the bottom traces which are for incident microwave frequencies of 63 and 302 $\mathrm{GHz}$, respectively. In order to substantiate this interpretation of the bump, we have examined its voltage position as a function of temperature.

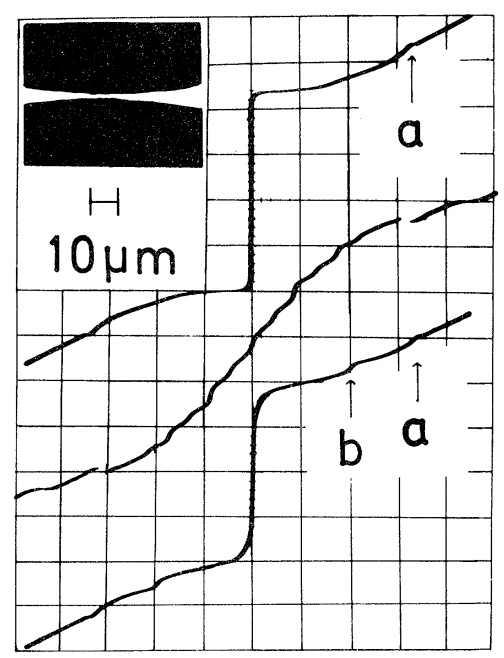

FIG. 1. $I V$ characteristic for an In weak link at 1.58 $\mathrm{K}$. Top curve, without applied microwaves; middle curve, exposed to $63-\mathrm{GHz}$ microwaves; bottom curve, exposed to $302-\mathrm{GHz}$ microwaves. Vertical scale 10 $\mu \mathrm{A} /$ div; horizontal scale $300 \mu \mathrm{V} /$ div. Inset, a photo of a typical weak link. 


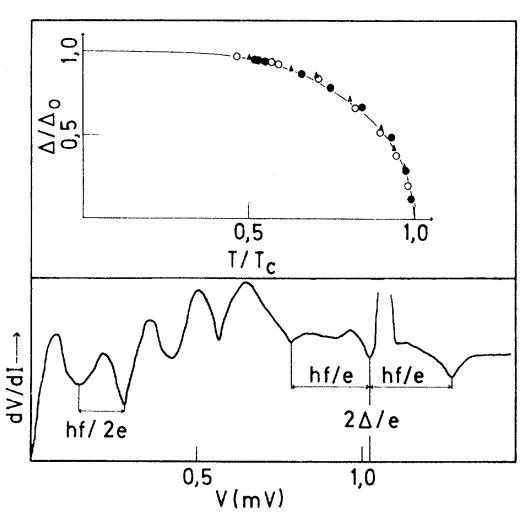

FIG. 2. Dynamic resistance as a function of the bias for an In weak link at $1.58 \mathrm{~K}$ exposed to $63-\mathrm{GHz}$ microwaves. The inset shows the measured energy gap versus the reduced temperature compared with the BCS theory for two In weak links (circles) and one Sn weak link (triangles).

Thus in Fig. 2 the dynamic resistance for the same weak link is shown for a microwave frequency of $63 \mathrm{GHz}$. The energy-gap structure is seen here more clearly. From a series of measurements of this type we are able to plot the variation of the energy gap with temperature as shown in the inset in Fig. 2. The points plotted refer to two In and one Sn weak links. For clarity some of our data have been omitted from the figure. The energy-gap structure has been observed in all our weak links. In plotting the points we have used only measured values, i.e., the measured temperature and voltage and the measured transition temperatures $T_{c}=3.40 \mathrm{~K}$ (In weak links) and $T_{c}=3.86$ (Sn weak link). The curve drawn in the inset is the BCS curve. The measured points follow this curve very closely, indicating the correctness of our interpretation of the bump.

(2) Microwave-assisted tunneling.-On the curve showing the dynamic resistance in Fig. 2, one notes (besides the Josephson step structure discussed below) an additional structure on either side of the gap. As the separation of the additional structure from the gap in both cases is very close to $h f / e$, it is tempting to interpret this structure in terms of microwave-assisted tunneling. For this reason we have examined the variation of the structure as a function of the incident microwave power and performed a comparison with the Tien-Gordon theory. ${ }^{9}$ Thus, in Fig. 3 we have plotted the variation of the dynamic conductance at the energy gap and at the additional structure as functions of the applied mi-

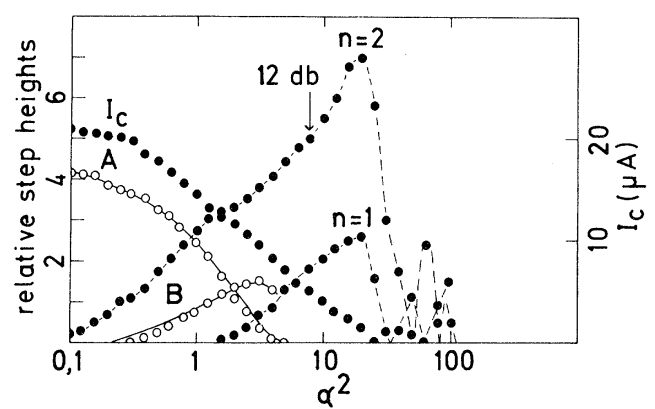

FIG. 3. Dynamic conductance peak at the energy gap $(A)$ and the first microwave-assisted step $(B)$ versus microwave power (open circles), compared with TienGordon expression. Also shown: $I_{c}$ (right-hand scale) and dynamic conductance peak for Josephson steps $n$ $=1,2$ (left-hand scale) versus microwave power (closed circles). $f=63 \mathrm{GHz}, T=1.58 \mathrm{~K}$.

crowave power at $63 \mathrm{GHz}$. The values of conductance were obtained by inverting the measured resistance values and subtracting a constant background term. For comparison we have also plotted the fully drawn curves, i.e., $J_{0}^{2}(\alpha)$ and $J_{1}^{2}(\alpha)$, based on the Tien-Gordon expression, for the gap and the first microwave-assisted peak, respectively. As is well known, ${ }^{10-12}$ the Tien-Gordon expression is valid for a tunnel junction whenever the spatial variation of the microwave field across the junction can be neglected. $J_{n}$ is the usual Bessel function and $\alpha=e V_{\mathrm{rf}} / h f$, where $V_{\mathrm{rf}}$ is interpreted as the $\mathrm{rf}$ voltage set up in the junction by the microwave field. It is apparent from Fig. 3 that the measured power dependence of the dynamic conductance at the gap and at the additional structure on either side of the gap very closely follows the Tien-Gordon expression for microwave-assisted tunneling. For this reason we interpret the additional structure to be the first microwave-assisted step. This kind of structure has only been distinctly observed when we measure the dynamic resistance. We have no clear evidence of higher-order microwave-assisted steps.

(3) Josephson steps.-Observations on Josephson steps in weak links have been reported earlier, notably by Gregers-Hansen et al., ${ }^{6}$ at 3 and $9 \mathrm{GHz}$ and at temperatures close to $T_{c}$. With the present weak links we easily observe the steps at 63 and $302 \mathrm{GHz}$ at low reduced temperature as shown by the characteristics reproduced in Fig. 1. Here the middle trace shows a series of $63-\mathrm{GHz}$ steps, while the lowest trace at the arrow $b$ shows a single Josephson step at 302 GHz. In both cases the steps occur at the right 
positions according to the relation $e V=h f / 2$, as shown in more detail in Fig. 2 which refers to the same weak link. We have further measured the power dependence of the Josephson dc current and the first six Josephson steps. However, for clarity we show in Fig. 3 only the measured power dependence for the dc current $I_{c}$ and for the first two steps $n=1$ and $n=2$. The points shown are the dynamic conductance taken from the dynamic resistance in the same way as was done for the microwave-assisted tunneling data in Sec. (2) and plotted on the same relative stepheight scale. From the fit between the microwave-assisted tunneling data and the Tien-Gordon expression the conversion from a decibel scale to an $\alpha$ scale is obtained. From Fig. 3 one notes that the zero for $I_{c}$ falls at an $\alpha^{2}$ value which is 4 times the $\alpha^{2}$ value for the zero for the microwave-assisted tunneling at the gap (curve $A$ ). This is in agreement with the $2 \alpha$ and the $\alpha$ appearing in the Bessel functions in the theoretical expressions for the microwave power dependence of, respectively, $I_{c}$ and the microwaveassisted tunneling in tunnel junctions and point contacts. Further, one notes that the power level at which the maximum for the first and second Josephson steps occurs corresponds to $\alpha=4.5$, which again corresponds to a microwave electric field of the order of $0.55 \mathrm{mV}$ in the thin-film weak link, indicating a good microwave coupling to the weak link. This is probably due to the shape of our weak links and their high resistance. Detailed measurements of this type have been performed on two In and one Sn weak link. The effect has been seen in most of our weak links.

One more feature of the Josephson-step data should be noted. As seen in Fig. 3, the Josephson step $n=2$ appears before and is larger than the step $n=1$. On account of this behavior we performed a series of measurements of the temperature dependence of the Josephson steps at a constant microwave power level, taken to be the one marked $12 \mathrm{~dB}$ in Fig. 3. From the measurements it follows that the magnitude of the steps shows an oscillating behavior, decreases with increasing temperature, and ultimately disappears just below the transition temperature. An analysis of our present data seems to indicate that if an even-numbered step occurs nearer to the energy gap than an odd-numbered step, then the even-numbered steps are enhanced. Conversely, if an odd-numbered step occurs nearer to the energy gap than an even-numbered step, then the odd-numbered steps are enhanced. This situa- tion is in many respects similar to the one described by Hamilton and Shapiro ${ }^{13}$ and by Buckner et al. ${ }^{14}$ which indicates that an experimental verification of the Riedel peak is possible also for a thin-film weak link. However our present data do not warrant a quantitative, detailed analysis. In summary, we have made thin-film weak links which have a very good coupling to the $\mathrm{mi}-$ crowave field because of their shape and high resistance. We have presented experimental evidence for the occurrence of an energy-gap structure and for microwave-assisted tunneling in thin-film weak links. Josephson steps have been observed at the high microwave frequencies 63 and $302 \mathrm{GHz}$. Further, from measurements of the power and temperature dependence of the Josephson steps it was also argued that the Riedel peak is observable in a thin-film weak link. Taken together with the recent ${ }^{8}$ observation of the quasiparticle-pair interference term, the $\cos \varphi$ term, in weak links it may be concluded that basically the thin-film weak links and the tunnel junctions show the same behavior.

We are very grateful to S. Shapiro for many discussions. Futher, we wish to thank Mr. P. H. Rasmussen and Mr. M. Bak for their assistance in taking some of the data and C. K. Bak for help with the electronic equipment.

${ }^{1}$ I. Giaever and H. R. Zeller, Phys. Rev. B $\underline{1}, 4278$ (1970).

${ }^{2}$ A. Longacre and S. Shapiro, Bull. Amer. Phys. Soc. $\underline{16}, 399$ (1971).

${ }^{3}$ O. Hoffmann Soerensen et al ., to be published.

${ }^{4}$ S. Shapiro, Phys. Rev. Lett. 11, 80 (1963).

${ }^{5}$ N. F. Pedersen et al., Phys. Rev. B $\underline{6}, 4151$ (1972).

${ }^{6}$ P. E. Gregers-Hansen et al., J. Low Temp. Phys. $\underline{7}$, 99 (1972). Recently these measurements have been extended to $35 \mathrm{GHz}$. Gregers-Hansen et al oclaim also to have observed subharmonics of the energy gap structure in their microbridges [P. E. Gregers-Hansen et al ., Phys. Rev. Lett. 31, 524 (1973)].

${ }^{7}$ A. A. Dayem and J. J. Wiegand, Phys. Rev. 155, 419 (1967).

${ }^{8}$ C. M. Falco, W. H. Parker, and S. E. Trullinger, Phys. Rev. Lett. 31, 933 (1973).

${ }^{9}$ P. K. Tien and J. P. Gordon, Phys. Rev. 129, 647 (1963).

${ }^{10}$ C. A. Hamilton and S. Shapiro, Phys. Rev. B $\underline{2}, 4494$ (1970).

${ }^{11}$ N. Z. Heidam, Phys. Lett. 35A, 378 (1971).

${ }^{12}$ M. R. Samuelsen, B. Kofoed, and O. Hoffmann Soerensen, Phys. Status Solidi (a) 11, K79 (1972).

${ }^{13}$ C. A. Hamilton and S. Shapiro, Phys. Rev. Lett. 26, 426 (1971).

${ }^{14}$ S. A. Buckner et al., Phys. Rev. Lett. 28, 150 (1972). 